\title{
Risk Factors for AMA Discharge From VA Inpatient Alcoholism Treatment Programs
}

\author{
Cynthia A. Loveland Cook, PhD,* Brenda M. Booth, Phd, $\dagger$ \\ Frederic C. Blow, Phd $\ddagger$ Kathleen A. McAleenan, BS, ${ }^{*}$ \\ AND JANICE Y. BunN, PhD $\dagger$ \\ *George Warren Brown School of Social Work, Washington University, St. Louis, Missouri; \\ †Center for Health Services Research, University of Iowa, Iowa City, Iowa; \\ łUniversity of Michigan Alcohol Research Center, Department of Psychiatry, Ann Arbor, Michigan
}

\begin{abstract}
This study was designed to identify those risk factors associated with discharge from inpatient alcoholism treatment against medical advice (AMA) and the underlying reasons for these discharges. Data on the characteristics of patients and their index hospitalization were obtained from the systematic review of medical records for 186 alcoholics who were discharged AMA and 201 alcoholics who completed treatment. Comorbid medical diagnosis reduced the risk of AMA discharge by one quarter, whereas court referral to treatment reduced the risk by one half. A college education, vocational or other training, being employed, and having a history of previous AMA discharges significantly increased the risk. The most common reasons for AMA discharge, as perceived by treatment providers, were psychosocial problems, difficulties in the treatment program, and lack of interest in treatment. The clinical implications of these findings for the inpatient treatment of alcoholics are discussed.
\end{abstract}

Keywords-alcoholism; inpatient treatment; attrition; AMA discharge.

\section{INTRODUCTION}

PrEMature TERMINATION of inpatient alcoholism treatment is an inefficient and ineffective use of the time, effort, cost, and resources expended to treat alcoholics. At the same time, early attrition often has adverse effects on patients, their families, and even treatment providers (Albott, 1982; Bowen \& Androes, 1968; Keil \& Esters, 1982). An exacerbation of alcohol use and

This study was supported by Grant R01-AA08732 (Booth, Cook, and Blow) from the National Institute of Alcohol Abuse and Alcoholism. We extend our appreciation to Pam Langefeld and Carl Langefeld for their contributions to this study.

Requests for reprints should be addressed to Cynthia A. Loveland Cook, PhD, Health Services Research \& Development (11H), VA Medical Center, 1481 West 10th Street, Indianapolis, IN 46202. alcohol-related problems (e.g., employment, health, interpersonal relationships) can follow premature discharge from treatment (Baekeland \& Lundwall, 1975; Bean \& Karasievich, 1975; Tomsovic, 1970) with eventual readmission. In a recent study conducted for the National Association of Addiction Treatment Providers, nearly one half of those patients whose treatment lasted 7 days or fewer were readmitted within the following year for chemical dependency relapse, psychiatric disorders, or other related conditions (Medstat Systems, Inc., 1991).

Varying rates of discharge against medical advice (AMA) have been reported. In a recent study of veterans who received inpatient alcoholism treatment in the Department of Veterans Affairs (VA), 1 out of 10 left AMA (Booth, Cook, \& Blow, 1992). Earlier re- 
search reported inpatient dropout rates ranging from $13.7 \%$ to $39.2 \%$ (Baekeland \& Lundwall, 1975; Beck, Shekin, Fraps, Borgmeyer, \& Witt, 1983; Knox, 1972). Recognizing that the early identification of alcoholics at risk for AMA discharge allows for the provision of clinical intervention to forestall such discharges, several studies have focused on those patient characteristics associated with premature termination from treatment. Booth et al. (1992) found that alcoholics with comorbid diagnoses of schizophrenia, other psychotic illnesses, and personality disorders were more likely to have AMA discharges than patients with other comorbid psychiatric diagnoses or none at all. Other risk factors associated with early attrition include age (Keil \& Esters, 1982; Schofield, 1978), personality factors (Jones, 1985; Sladen \& Mozdzierz, 1985), history of previous AMA discharges (Booth et al., 1992; Keil \& Esters, 1982), severity of alcoholism (Miller, Pokorny, \& Hanson, 1968), socioeconomic status (Heineman, Moore, \& Gurel, 1976; Pekarik \& Zimmer, 1992), and social stability (Baekeland \& Lundwall, 1975).

Most studies on AMA discharges were conducted nearly two decades ago when inpatient treatment was generally characterized by 60 - to 90 -day lengths of stay. Not only were small sample sizes used from single treatment sites, they also tended to focus exclusively on patient-specific characteristics (Pekarik \& Zimmer, 1992). Few studies assessed the relationship of AMA discharges to the characteristics of alcoholism treatment environments, and even fewer studies examined the underlying reasons for AMA discharges as perceived by patients or staff. However, it is reasonable to assume that ineffective treatment modalities, patient/ staff conflict, the pressure of social problems, unrealistic expectations of clinical interventions, and similar reasons contribute to early attrition from treatment.

The major purposes of this study were to determine which risk factors are associated with AMA discharge from inpatient alcoholism treatment and to describe primary reasons for these types of discharge. This study provides information that can help clinicians identify those patients who are most at risk for AMA discharge, thereby providing the opportunity to initiate preventive measures to decrease unnecessary attrition. The successful implementation of preventive measures can enhance not only positive health and mental health outcomes in these patients but also the efficient and cost-effective use of limited treatment resources. Furthermore, from a quality assurance perspective, information on the most common reasons for AMA discharge can serve as a focus for program-level assessment, intervention, and monitoring.

\section{METHOD}

A sample of 186 alcoholics was selected from a computer-generated list of AMA discharges that oc- curred between 1987 and 1989 from two inpatient alcoholism treatment programs. AMA discharges were operationally defined as individuals who never returned from an authorized pass, those who simply walked off the unit, and those who left after discussing termination of their treatment with one or more treatment providers. For purposes of comparison, 201 alcoholics who completed treatment during the same time period in the same programs were randomly selected for inclusion.

\section{Sample}

Both groups of patients were treated in one of two inpatient alcoholism treatment programs. One program was housed in a 307-bed VA medical center that predominantly treats individuals from rural communities, whereas the other program was located in a 500-bed university-affiliated VA medical center that provides services to individuals living in a large urban area.

The majority of patients in this study were unmarried White alcoholics with a high school education or less. Over one half were employed. Their ages ranged from 19 to 73 years, with a mean age of 41.8 years. Because few female veterans receive inpatient alcoholism treatment in the VA (Booth et al., 1992), the sample in this study included only men.

\section{Data Collection}

Each patient's medical record was reviewed by an interviewer who received intensive training on review procedures and protocol. A written training manual was developed that specifically described the types of information needed from these records.

Two types of information were obtained, including characteristics of patients and their index hospitalization for alcoholism treatment. In the former, data included sociodemographic information (e.g., age, marital status, education, race, employment status) and preadmission history (e.g., alcohol use, previous AMA discharges, suicidal and violent behavior). Data related to the index hospitalization focused on length of stay in the treatment program; comorbid medical and psychiatric diagnoses; and, for patients leaving AMA, the primary reason underlying their discharge from treatment.

The primary reason for each AMA discharge was coded for content by three trained coders. Only content codes agreed upon by at least two out of three coders were used in the data analyses. Using a total of 13 content categories, $92.6 \%$ agreement was reached for this variable. Comparisons were then made between alcoholics with codable and noncodable responses by other variables in the study. Significant differences were not found. 
TABLE 1

Sociodemographic Characteristics of Alcoholics Discharged AMA and Alcoholics Completing Inpatient Alcoholism Treatment

\begin{tabular}{lcc}
\hline $\begin{array}{l}\text { Sociodemographic } \\
\text { Characteristic }\end{array}$ & $\begin{array}{c}\text { AMA } \\
(n=186)\end{array}$ & $\begin{array}{c}\text { Non-AMA } \\
(n=201)\end{array}$ \\
\hline Age (in years) & 41.4 & 42.2 \\
Employment status (\% employed) & 61.8 & $52.8^{*}$ \\
Marital status (\% married) & 32.4 & 30.0 \\
Race (\% non-White) & 13.2 & 14.1 \\
Education (\% high school or less) & 56.0 & $67.5^{\star *}$ \\
\hline
\end{tabular}

Note. Levels of significance were determined using chi-square tests of independence.

${ }^{\star} p<.07 .{ }^{* *} p<.05$.

\section{Data Analyses}

Differences between the AMA and non-AMA groups were examined using either chi-square tests of independence or Student $t$ tests, depending on the dichotomous or continuous nature of the variables being investigated. To identify risk factors associated with AMA discharge, those independent variables with strong bivariate associations with AMA discharge were then included in a logistic regression model to determine their respective odds ratios. The latter approximates the likelihood of AMA discharge in the presence of the independent variables as risk factors (Fletcher, Fletcher, \& Wagner, 1988). The reasons associated with AMA discharge from alcoholism treatment programs, on the other hand, were determined using frequency distributions.

\section{FINDINGS}

As shown in Table 1, alcoholics who left AMA and those who completed treatment had similar demographic characteristics (e.g., age, race, marital status). However, those in the AMA group had more education than those in the non-AMA group. Only $56.0 \%$ of the AMA group had a high school education or less, whereas this level of education characterized $67.5 \%$ of the comparison group. The AMA group also was more likely to be employed.

\section{Comparison of Alcoholics Discharged AMA and Those Completing Treatment}

The preadmission history of alcohol use between the AMA and non-AMA groups differed in several ways (see Table 2). The onset of frequent and/or problem

TABLE 2

Comparison of Preadmission History and Hospitalization of AMA and Non-AMA Patients

\begin{tabular}{|c|c|c|}
\hline Characteristic & $\begin{array}{c}\text { AMA } \\
(n=186)\end{array}$ & $\begin{array}{c}\text { Non-AMA } \\
(n=201)\end{array}$ \\
\hline \multicolumn{3}{|l|}{ Preadmission history } \\
\hline \multicolumn{3}{|l|}{ Alcohol use } \\
\hline Quantity (drinks/day) & 25.4 & $21.3^{*}$ \\
\hline Age of onset (years) & 20.3 & $17.7^{* *}$ \\
\hline Days abstinent prior to admission & 3.5 & 5.1 \\
\hline \multicolumn{3}{|l|}{ History of high-risk behaviors } \\
\hline Violence & 14.7 & 16.0 \\
\hline Suicide attempts & 9.8 & 12.9 \\
\hline \multicolumn{3}{|c|}{ Utilization of alcoholism treatment services } \\
\hline Past inpatient alcoholism treatment & 74.1 & $60.8^{\star \star}$ \\
\hline Previous AMAs & 24.7 & $11.0^{* \star *}$ \\
\hline \multicolumn{3}{|l|}{ Hospitalization } \\
\hline \multicolumn{3}{|l|}{ Referral sources } \\
\hline Self-referral & 78.0 & 72.6 \\
\hline Court referral & 7.0 & $14.4^{*}$ \\
\hline Other referral & 20.4 & 22.9 \\
\hline \multicolumn{3}{|l|}{ Patient characteristics } \\
\hline Comorbid psychiatric diagnoses & 16.1 & 15.4 \\
\hline Comorbid medical diagnoses & 57.0 & $86.1 * * *$ \\
\hline Suicidal at admission & 7.6 & 6.0 \\
\hline \multicolumn{3}{|l|}{ Inpatient stay } \\
\hline Mean length of stay (in days) & 8.3 & $22.2^{* * *}$ \\
\hline Length of stay less than $24 \mathrm{hr}$ & 19.9 & $1.0^{* \star \star}$ \\
\hline Discharge plan (\% received) & 38.6 & $93.0^{* * *}$ \\
\hline
\end{tabular}

Note. Levels of significance were determined using chi-square tests of independence or Student $t$ tests depending on the categorical or continuous nature of the variable.

${ }^{\star} p<.05 .{ }^{\star \star} p<.01 .{ }^{\star \star \star} p<.001$. 
drinking occurred at a later age in the AMA group (20.3 years) than in the comparison group (17.7 years). Although there was little difference in days of abstinence prior to admission, the AMA group consumed larger quantities of alcoholic beverages on a daily basis than the non-AMA group $(M \mathrm{~s}=25.4$ and 21.3 drinks per day, respectively). At the same time, the AMA group was less likely to be referred for treatment by the court system than those alcoholics who completed treatment $(7.0 \%$ and $14.4 \%$, respectively). Few alcoholics in either group engaged in violent or suicidal behavior prior to admission.

Alcoholics who left AMA were more likely to have been hospitalized in the past for alcoholism treatment than those who completed treatment $(74.1 \%$ and $60.8 \%$, respectively) and were also more likely to have had previous AMA discharges $(24.7 \%$ and $11.0 \%$, respectively). As expected, the AMA group had considerably shorter lengths of stay in the treatment program than the comparison group ( $M \mathrm{~s}=8.3$ and 22.2 days, respectively). Although there was no significant seasonal variation in the month of admission or discharge, the AMA patients were more likely to be discharged in the afternoon than those patients who completed treatment, $t(342)=-3.85, p<.001$.

Excluding diagnoses for minor audiology, ophthalmology, dental, and dermatology disorders, $72.1 \%$ of the entire sample had comorbid medical diagnoses. A significantly smaller proportion of the AMA patients had comorbid medical diagnoses $(57.0 \%)$ compared to the non-AMA patients $\left(86.1 \% ; \chi^{2}=40.6, d f=1\right.$, $p<.001)$. Nearly one fifth of both groups had at least one comorbid psychiatric disorder in addition to their primary alcohol-related diagnosis.

\section{Risk Factors Associated With AMA Discharges}

Using a logistic regression model, the next analysis determined which factors posed a risk for AMA discharge from inpatient alcoholism treatment programs. The dependent measure was a dichotomous variable that compared patients discharged AMA to those completing treatment. In addition to type of inpatient alcoholism treatment program, the independent variables included those factors that had strong bivariate relationships with the dependent measure (see Tables 1 and 2). Age at onset of drinking and number of daily drinks before admission were included as continuous variables. Using high school or less as the reference group, two education variables were created: (a) college and (b) vocational or other training. Employment status, previous inpatient treatment, referral by the court, previous AMA discharge, and comorbid medical diagnoses were also coded as "yes/no" with "no" being the reference group.

The overall logistic regression model was significant at the .001 level $\left(\chi^{2}=64.20, d f=9\right)$. With the exception of previous inpatient admissions for alcoholism treatment, all the independent variables were retained in the model using a $p$-value cutoff of .10 (see Table 3). A comorbid medical diagnosis reduced the risk of AMA discharge to one quarter, whereas being referred to treatment by the court system reduced the risk by one half. Respondents with a history of previous AMA discharges were more than $2 \frac{1}{2}$ times as likely to leave early. The odds of AMA discharge increased twofold for college education and vocational or other training, and it increased $1 \frac{1}{2}$ times for employment.

\section{Characteristics of AMA Discharges}

Next, those patient and environmental factors perceived by staff to be the primary reasons underlying AMA discharge were assessed (see Table 4). Psychosocial problems were cited in over one third of all discharges, particularly interpersonal problems (e.g., spouse, family, friends; $10.5 \%$ ), socioeconomic problems (e.g., financial or employment problems; $9.9 \%$ ), and either health or mental health problems $(8.6 \%)$. Nearly one out of four alcoholics who left AMA had documented

TABLE 3

Odds Ratios for Risk Factors of AMA Discharge From Inpatient Alcoholism Treatment Programs

\begin{tabular}{lccc}
\hline Risk Factor & Odds Ratio & $\begin{array}{c}95 \% \text { Confidence } \\
\text { Interval }\end{array}$ & $p$ \\
\hline Employment & 1.69 & $1.01-2.82$ & .045 \\
College & 2.07 & $1.09-4.36$ & .027 \\
Vocational and other training & 2.29 & $1.27-4.13$ & .006 \\
Age of onset of drinking & 1.06 & $1.02-1.10$ & .002 \\
Previous AMA discharges & 2.55 & $1.32-4.94$ & .006 \\
Number of daily drinks before admission & 1.01 & $0.99-5.15$ & .068 \\
Referral by court & 0.46 & $0.20-1.06$ & .071 \\
Comorbid medical diagnoses & 0.24 & $0.13-0.43$ & .000 \\
\hline
\end{tabular}

${ }^{a}$ The odds ratios were derived from the parameter estimates of a stepwise multiple logistic regression model that forced treatment program into the equation. 
TABLE 4

Primary Reason for AMA Discharges From inpatient Alcohollsm Treatment Program $(n=186)$

\begin{tabular}{lrr}
\hline Reason & Percent & $n$ \\
\hline Psychosocial problems & & \\
Employment/financial & 9.9 & 16 \\
Interpersonal & 10.5 & 17 \\
Housing & 1.9 & 3 \\
Legal & 2.5 & 4 \\
Health/mental health & 8.6 & 14 \\
Other & 4.3 & 7 \\
Problems in program & & \\
Conflict with staff and/or patients & 4.9 & 8 \\
Not following rules of program & 9.9 & 16 \\
Dissatisfied with care & 8.6 & 14 \\
Wants treatment elsewhere & 1.9 & 3 \\
Lack of interest & & \\
$\quad$ Not interested in receiving treatment & 15.4 & 25 \\
Coerced into treatment program & 2.5 & 4 \\
Other & & \\
$\quad$ No reasons given & 19.1 & 31 \\
\hline
\end{tabular}

problems with the treatment program. Failure to follow treatment program rules (e.g., not drinking alcoholic beverages while enrolled in treatment and not attending scheduled meetings) and dissatisfaction with care was common ( $9.9 \%$ and $8.6 \%$, respectively). Last of all, $15.4 \%$ of the alcoholics discharged AMA were perceived by staff to lack interest in treatment for alcoholism.

One out of five $(\mathbf{2 0 . 1 \%})$ of the AMA group left within $24 \mathrm{hr}$ of admission. Compared to those AMA alcoholics with longer lengths of stay, these individuals were more likely to have suicidal ideations upon admission and to have refused treatment during this hospital stay (see Table 5). They also werc less likcly to have legal and financial problems or to have had previous admissions for alcoholism treatment.

An important component of treatment involves discharge planning to enhance posthospital functioning through needs assessment and the provision of tangible services in the community. In this study, $38.6 \%$ of the AMA group received discharge planning, whereas this service was provided to $93.0 \%$ of the alcoholics who completed treatment $\left(\chi^{2}=128.8, d f=1, p<.001\right)$.

\section{DISCUSSION}

This study used a large sample of alcoholics who were discharged AMA and a comparison group of alcoholics who completed treatment to determine which factors were associated with early attrition from inpatient alcoholism treatment programs. From a historical perspective, those in the AMA group were more likely to have an earlier onset of problem drinking and previ- ous AMA discharges from alcoholism treatment programs that their non-AMA counterparts. Nearly one fifth of this group left the hospital within $24 \mathrm{hr}$ of admission. The AMA group also was less likely to have been referred for treatment by the court system or to have comorbid medical diagnoses. As perceived by staff, the most common reasons for AMA discharges were psychosocial problems, difficulties with the treatment program, and lack of interest in treatment.

Because previous AMA discharge from inpatient alcoholism treatment was an important risk factor for current AMAs in this study and was significantly associated with AMA discharges in other studies (Booth et al., 1992; Keil \& Esters, 1982), it is evident that some alcoholics are becoming "revolving door" patients. Repeated admissions and discharges reflect not only the inability of some alcoholics to benefit from available treatment but also difficulties in the provision of appropriate care for this special population. In either case, AMA discharges deprive other alcoholics who might benefit from inpatient treatment of this important resource, particularly in programs with long waiting lists.

An important risk factor for AMA discharge was referral for treatment by sources other than the court system. As Pekarik and Zimmer (1992) emphasized, a powerful motivation to continue treatment is the avoidance, reduction, or suspension of a jail sentence that is made contingent on the completion of treatment. On a similar note, alcoholics in the AMA group were also less likely to have comorbid medical diagnoses. Alcoholics with medical problems may be more dependent on program staff for assistance with and treatment of their health problems. Likewise, caregivers may withdraw support for medically ill alcoholics who want to withdraw prematurely from treatment.

TABLE 5

Comparison of AMA Group with Less than 24-hr Stay Versus Longer Lengths of Stay

\begin{tabular}{lcc}
\hline & $\begin{array}{c}\text { Less Than } \\
24 \mathrm{hr} \\
(n=37)\end{array}$ & $\begin{array}{c}\text { More Than } \\
24 \mathrm{hr} \\
(n=147)\end{array}$ \\
Characteristics & 59.5 & $77.4^{*}$ \\
\hline $\begin{array}{l}\text { Previous inpatient } \\
\text { alcoholism treatment }\end{array}$ & 16.2 & $5.4^{\star}$ \\
$\begin{array}{l}\text { Suicidal ideations upon } \\
\text { admission }\end{array}$ & 29.7 & $8.1^{\star \star *}$ \\
$\begin{array}{l}\text { Refused to participate in } \\
\text { current treatment }\end{array}$ & 8.1 & $30.9^{* *}$ \\
$\begin{array}{l}\text { Legal problems } \\
\text { Financial problems } \\
\text { Discharge planning before } \\
\text { left program }\end{array}$ & 10.8 & $33.6^{\star *}$ \\
\hline
\end{tabular}

Note. Levels of significance were determined using chi-square tests of independence.

${ }^{\star} p<.05 .{ }^{\star *} p<.01 .{ }^{* *} p<.001$ 
Although earlier studies speculated that inpatient treatment was threatening or anxiety provoking for alcoholics with characterological and emotional problems (Booth et al., 1992; Krasnoff, 1976; Schofield, 1978), this study found no association between documented comorbid psychiatric diagnoses (including personality and psychotic disorders) and AMA discharge. Because these data are based on clinical diagnoses, it is possible that comorbid mental health disorders were underdiagnosed on these units, particularly for the AMA group whose short length of stay precluded in-depth assessment. It is reasonable to expect that the mental health of alcoholics will affect their response to and interactions with their treatment programs. Some will find the rules and regulations of the treatment milieu intolerable. Leaving AMA may be an impulsive and even hostile gesture for others, arising out of conflict with treatment staff. In this study, nearly $25 \%$ of the AMA group were perceived as having problems with the treatment program, including conflict with staff and not following rules.

For patients leaving AMA within $24 \mathrm{hr}$ of admission, both patient motivation and admitting practices must be assessed further. In this study, nearly one third of these alcoholics $(\mathbf{2 9 . 7 \%})$ refused to be involved in the treatment program altogether. Perhaps lack of motivation to engage in treatment was not adequately assessed by the admitting staff, or perhaps the motivation expressed by patients who were intoxicated on admission substantially changed with sobriety.

Several factors may contribute to the low rate of discharge planning $(38.5 \%)$ for the alcoholics who left AMA. First, because some patients simply walked off the unit or left the program while on pass, there may have been limited opportunity for discharge planning. Second, as proposed by Glick, Braff, Johnson, and Showstack (1981), it is possible that some treatment providers may withhold assistance with discharge medications and/or follow-up referrals based on provider beliefs that alcoholics who leave AMA act against their best interests. In either case, it is critical that the posthospital needs of these individuals be addressed in an effort to better influence outcomes, despite their limited involvement in treatment.

Because data on the underlying reasons for AMA discharges were obtained from medical record reviews, interpretation of the findings must consider that these data reflect the perception of treatment providers. Keeping this in mind, nearly two fifths of the alcoholics who left AMA had psychosocial problems reported as the primary reason for their early attrition from the program, particularly interpersonal difficulties. Earlier studies found that social isolation and family problems were associated with dropping out of treatment (Baekeland \& Lundwall, 1975; Pisani \& Motansky, 1970; Zax, Marsey, \& Biggs, 1961). From a treatment perspective, early assessment and intervention with psychosocial problems may reduce unnecessary attrition. For example, psychosocial problems of an emotional nature, such as anxiety or depression, can be identified and treated as co-occuring diagnoses. Early interventions can also be focused on helping patients with their employment problems, financial difficulties, and interpersonal problems.

Difficulties with the treatment program were described as a common reason for AMA discharge. Although research has tended to focus almost exclusively on the characteristics of patients who leave AMA, it is reasonable to assume that problems with the treatment environment also contribute to early attrition. Treatment modalities, admission criteria, and treatment staff are all factors that can influence the rate of AMA discharges. From the perspective of treatment matching, perhaps giving alcoholics a choice of treatment modalities will decrease AMA rates (Kissin, Platz, \& Su, 1971).

In summary, this study provided an initial glimpse of patient-specific and environmental factors associated with AMA discharges from inpatient alcoholism treatment. Future research might focus on the identification of a high-risk profile of patients who leave AMA so that a screening instrument can be developed for early detection and intervention. Furthermore, educating staff about the characteristics of this population can help them identify alcoholics at risk for AMA discharge so that they can intervene earlier and more effectively. Aspects of the therapeutic environment could also be adapted or individualized to reduce the probability that these patients will drop out of treatment. Furthermore, it is critical that the monitoring of AMA discharges be an integral component of quality assurance activities in alcoholism treatment.

\section{REFERENCES}

Albott, W.L. (1982). Dropouts from an inpatient treatment program for alcoholics. The International Journal of the Addictions, 17, 199-204.

Baekeland, F., \& Lundwall, L. (1975). Dropping out of treatment: A critical review. Psychological Bulletin, 82, 738-783.

Bean, K.L, \& Karasievich, G.O. (1975). Psychological test results at three stages of inpatient alcoholism treatment. Journal of Studies on Alcohol, 36, 838-852.

Beck, N.C., Shekin, W., Fraps, C., Borgmeyer, A., \& Witt, A. (1983). Prediction of discharges against medical advice from an alcohol and drug misuse treatment program. Journal of Studies on Alcohol, 44, 171-180.

Booth, B.M., Cook, C.A.L., \& Blow, F.C. (1992). AMA discharges from alcoholism treatment: Role of psychiatric comorbidity. Hospital and Community Psychiatry, 43, 730-731.

Bowen, W.T., \& Androes, L.R. (1968). A follow-up study of 79 alcoholic patients: 1963-1965. Bulletin of the Menninger Clinic, 32, 26-34.

Fletcher, R.H., Fletcher, S.W., \& Wagner, E.H. (1988). Clinical epidemiology. Baltimore: Williams \& Wilkins. 
Glick, I.D., Braff, D.L., Johnson, G., \& Showstack, J.A. (1981). Outcome of irregularly discharged psychiatric patients. American Journal of Psychiatry, 138, 1472-1476.

Heineman, E., Moore, B., \& Gurel, M. (1976). Completion of termination of alcoholism treatment: Toward the development of a predictive index. Psychological Reports, 38, 1340-1342.

Jones, J.W. (1985). Predicting patients' withdrawal against medical advice from an alcoholism treatment center. Psychological Reports, 57, 991-994.

Keil, T.J., \& Esters, R.A. (1982). treatment dropouts: The effect of client and ecological variables The International Journal of the Addictions, 17, 95-108.

Kissin, B., Platz, A., \& Su, W.H. (1971). Selective factors in treatment choice and outcome in alcoholics. In N.K. Mello \& J.H. Mendelson (Eds.), Recent advances in studies of alcoholism: An interdisciplinary symposium (pp. 781-802). Rockville, MD: US National Institute of Alcohol Abuse and Alcoholism.

Knox, W.J. (1972). Four-year follow-up of veterans treated on a small alcoholism ward. Quarterly Journal of Studies on Alcohol, 33, 105-110.

Krasnoff, A. (1976). Differences between alcoholics who complete or withdraw from treatment. Journal of Studies on Alcohol, 37, 1666-1671.

Medstat Systems, Inc. (1991). Treatment is the answer: $A$ white pa- per on the cost effectiveness of alcoholism and drug dependency treatment. Laguna Hills, CA: National Association of Addiction Treatment Providers.

Miller, B., Pokorny, A., \& Hanson, P. (1968). A study of dropouts in an inpatient alcoholism treatment program. Diseases of the Nervous System, 29, 91-99.

Pekarik, G., \& Zimmcr, L. (1992). Rclationship of client variables to continuance in five types of alcoholism treatment settings. $A d$ dictive Behaviors, 17, 105-115.

Pisani, V.D., \& Motansky, G.U. (1970). Predictors of premature termination of outpatient follow-up group psychotherapy among male alcoholics. International Journal of the Addictions, $\mathbf{5}$, 731-737.

Schofield, L.J. (1978). Internal-external control and withdrawal AMA from an alcohol rehabilitation program. Journal of Clinical Psychology, 34, 571-573.

Sladen, B.J., \& Mozdzierz, G.J. (1985). An MMPI scale to predict premature termination from inpatient alcohol treatment. Journal of Clinical Psychology, 41, 855-862.

Tomsovic, M. (1970). A follow-up study of discharged alcoholics. Hospital and Community Psychiatry, 21, 94-97.

Zax, M., Marsey, R., \& Biggs, C.F. (1961). Demographic characteristics of alcoholic outpatients and tendency to remain in treatment. Quarterly Journal of Studies on Alcohol, 23, 634-639. 